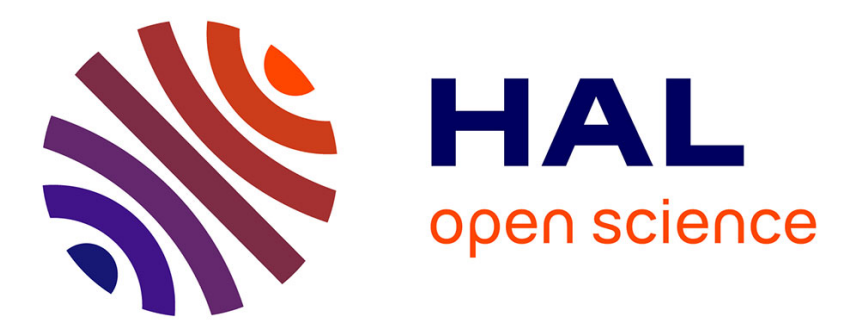

\title{
5-axis Direct Machining of Rough Clouds of Points
}

Almohanad Makki, Christophe Tournier, François Thiebaut, Claire Lartigue, Charyar Mehdi-Souzani

\section{To cite this version:}

Almohanad Makki, Christophe Tournier, François Thiebaut, Claire Lartigue, Charyar Mehdi-Souzani. 5-axis Direct Machining of Rough Clouds of Points. CAD'10, Jun 2010, Dubai, United Arab Emirates. pp.591-600, 10.3722/cadaps.2010.591-600 . hal-00577730

\section{HAL Id: hal-00577730 \\ https://hal.science/hal-00577730}

Submitted on 17 Mar 2011

HAL is a multi-disciplinary open access archive for the deposit and dissemination of scientific research documents, whether they are published or not. The documents may come from teaching and research institutions in France or abroad, or from public or private research centers.
L'archive ouverte pluridisciplinaire HAL, est destinée au dépôt et à la diffusion de documents scientifiques de niveau recherche, publiés ou non, émanant des établissements d'enseignement et de recherche français ou étrangers, des laboratoires publics ou privés. 


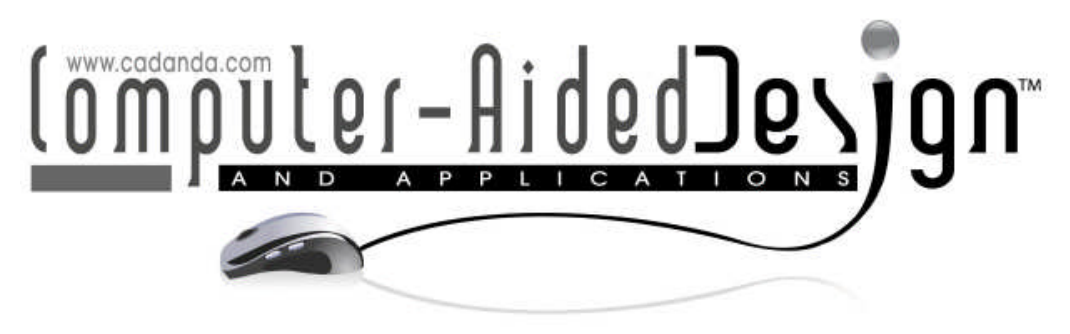

\title{
5-axis Direct Machining of Rough Clouds of Points
}

\author{
Mohanad Makki, Christophe Tournier, François Thiebaut, Claire Lartigue and Charyar Souzani \\ LURPA, ENS Cachan, Université Paris Sud 11 - France, name@lurpa.ens-cachan.fr
}

\begin{abstract}
Direct duplication of free-forms by 5-axis machining consists in the machining of rough clouds of points without surface reconstruction, with the aim at minimizing deviations between the original object and its copy. As discrete 5-axis machining is concerned, the surface is machined area by area with various tool orientations. However, the quality of the machine tool geometry may be source of overlapping errors at area junctions. In the paper, a method for direct duplication by discrete 5axis machining is proposed. Data segmentation into areas relies on the global visibility of the points, including the visibility of the point cloud by the machine tool. A criterion accounting for the minimization of the overlapping area is also introduced to ensure the quality of the machined object.
\end{abstract}

Keywords: freeform prototyping, point clouds, 5-axis milling, geometrical deviations. DOI: $10.3722 /$ cadaps.2010.591-600

\section{INTRODUCTION}

3D digitizing of part surfaces using non contact sensors is now a widespread process in industry as it delivers an image of the surface as large sets of points in a relatively short time. Among the numerous applications, physical object duplication permits the recreation of hand-made objects or prototypes. Most methods rely on a Reverse Engineering (RE) process. For such methods, the objective consists in converting the discrete data into a usable surface model. RE process consists of three main steps: (1) data capture using a 3D digitizing system, feature extraction, surface reconstruction or CAD modelling [13]. The CAD model is afterwards used for tool path generation or rapid prototyping. Despite the advances in CAD modelling of discrete data, the step of RE is not automatic, and generally time consuming. The quality of the resulting surface strongly depends on the possibilities offered by the Software: extraction of style lines, data segmentation and precision of surface fitting for complex objects [3]. Moreover, the CAD modelling step involves the smoothing of small details not always expected [7]. Hence, object duplication process would take advantage in direct tool path calculation from discrete data points. In this context, the paper deals with the implementation of a functional chain of object duplication by machining, from the 3D digitizing process to the actual surface machining in 5 axes. Direct duplication using 5 -axis milling is a process for which the step of CAD modelling is removed. Therefore, CNC tool path generation is directly performed from the cloud of points collected during the 3D digitizing stage. In the literature, only a few methods investigate direct machining of digitized points according to various sensor configurations. Indeed, in this case, collected points define a large cloud of points, non-ordered, dense, noisy, and inhomogeneous. Most 
methods take advantages of the digitizing lines [5], [10] when they exist or reconstruct a pseudocontinuity through a meshing or a cell-net built from the point cloud [3]. More recently, Teng et al. [12] introduced the concept of area by area machining of complex parts in order to increase machining efficiency. The point cloud is segmented in function of the geometric complexity of the points defined considering possible gouging. Points with the same complexity are grouped together to form distinct machining areas. However, the point cloud proposed for illustration comes from the sampling of a CAD model, and not from an actual digitizing.

Only a few approaches address the problem of discrete 5-axis machining directly from the cloud of points without surface or meshing reconstruction. Discrete 5-axis machining is similar to area by area machining as it consists in defining group of points accessible by the same tool orientation. For each orientation, the portion of surface accessible by the tool (most generally a ball-end cutter tool) is machined using 3-axis machining algorithms [7]. Such algorithms have been tested with success in a previous study, highlighting that the surface accuracy obtained is close to, even better than, that obtained using the classical RE method [7]. Basically, the main issue of discrete 5-axis machining can be formulated as follows: given the point cloud and the 5-axis machine tool, find the minimum number of tool orientations so that all the points are accessible. However, although nowadays machine tools have high-performance, machine tool geometrical errors still remain (axis straightness, axis perpendicularity...). Those errors induce re-positioning errors on the machine tool thus involving geometrical deviations on the machined part at the area junctions (Fig. 1). Therefore, overlaps must be limited.

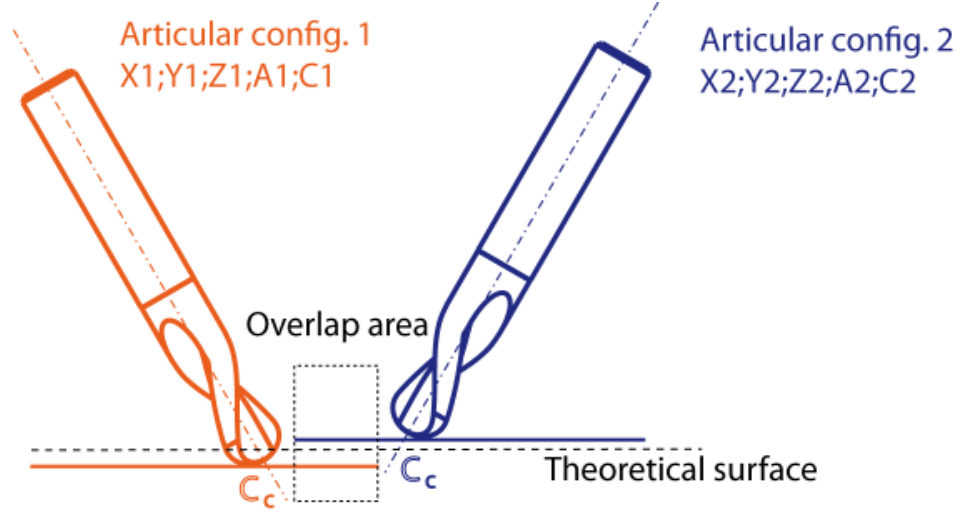

Fig. 1: Geometrical errors due to tool re-positioning.

The method proposed in the paper consists in data segmentation so that the overlapping length is minimal in order to ensure visual as well as geometrical quality of the object copy. Data segmentation relies on the point cloud partitioning according to visibility criteria. Accordingly, we introduce the concept of global visibility including the tool accessibility (or visibility), the non gouging condition, and the feasibility of the machining on the considered machine tool.

\section{GLOBAL VISIBILITY CALCULATION}

From a geometrical point of view the most important issue is to ensure the feasibility of the surface machining on the machine tool. That means, how defining the initial part set-up so that the part machining will be complete? In this direction, we have to account for the machine tool working space must be taken into account. Furthermore, interferences, i.e. local gouging as well as global interferences between the tool and its cutting environment (fixtures, tool holder, etc.), have to be avoided. The effective issue is the visibility calculation from direct raw data due to the absence of continuity and the lack of data structuring. The global visibility method consists of 3 mains steps based on the concept of the Gaussian sphere which provides the space discretization: the machine visibility, the tool visibility and the global visibility. 
As part of the global visibility calculation, the evaluation of the local normal to the discrete data is an essential issue. Indeed, due to the absence of data continuity, the normal can not be directly calculated and must be estimated. The next section is dedicated to the method developed for normal calculation on raw data points.

\subsection{Normal Calculation}

In the literature, methods of normal calculation from raw data can be classified into three main families. Methods of the first family generally consist in fitting least-square planes to sets of data points, the normal of the plane defining the local normal to the data. Points are grouped according to form deviation and local curvature criteria [2][8]. Such methods are well adapted to slender surfaces but not to complex surfaces with high curvature. The correct orientation of the normal is also tricky. The second family gathers methods, which locally associate curves [9], or surfaces [14] to sets of points. Methods give good results when the cloud of points is homogeneous and slightly noisy. As for the previous family, the determination of the correct normal orientation is a difficulty. The last family concerns methods associating a surface meshing to the points [6]. The normal thus corresponds to the normal to each facet. These methods ensure a correct normal orientation. Nevertheless, when applied to raw data they are complex and time consuming.
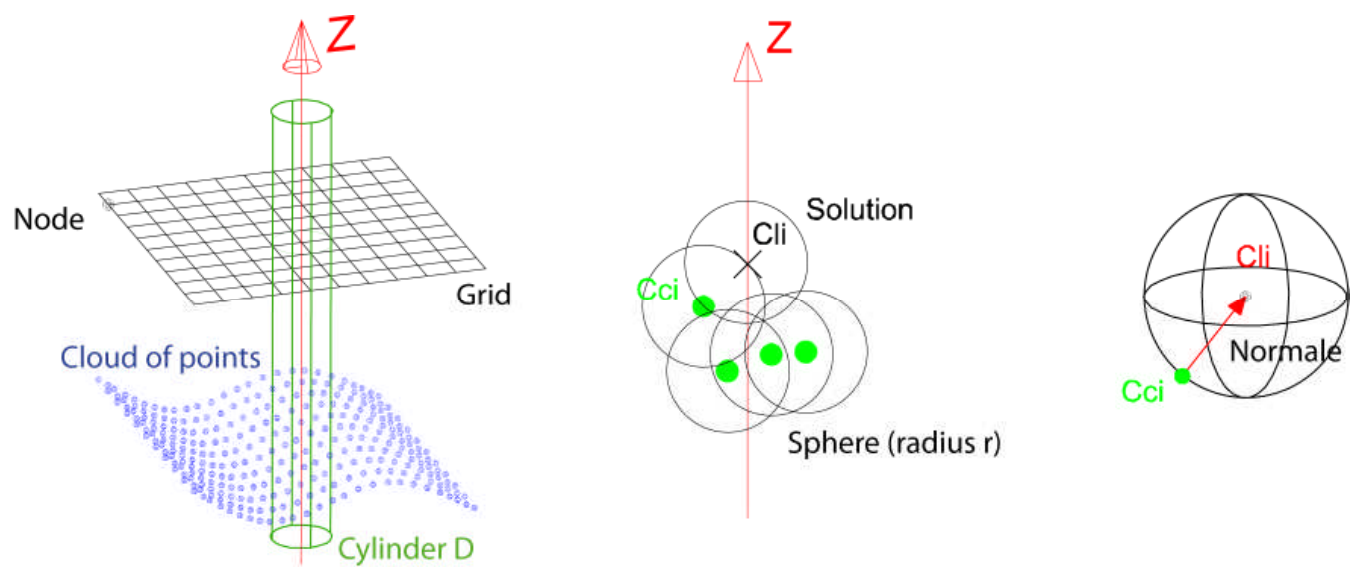

Fig. 2: Normal calculation by sphere throwing.

In the paper, we propose a method based on the classical concepts of ball-end milling tool positioning on a surface. Let us consider a rough cloud of points, and a given direction $Z$ corresponding to the throwing direction (Fig. 2). A grid of points (Xdi, Ydi) is defined in the perpendicular plane to Z; a line parallel to $\mathrm{Z}$ is built at each node of the grid, Ndi. A sphere, the radius of which is $\mathrm{r}$, is thus thrown along each line. As the sphere touches the cloud of points at a point Cci, the normal is defined as follows:

$$
\vec{n}=\overrightarrow{C c i C l i}
$$

In practice, the calculation is restrained to the cloud portion defined by the cylinder of radius $\mathrm{R}$, passing through Ndi, which axis is Z. The calculation thus consists in calculating the intersections between all the spheres centred at each point of the cylindrical portion and the considered line. For the intersection giving the maximal altitude $\mathrm{Zi}$, the centre of the sphere Cli is defined by (Xdi,Ydi,Zi), and the position of the contact point Cci is marked. Therefore, the normal at the point Cci is calculated using Eqn. (2.1).

Remark1: with such a method, the normal is always oriented towards the centre of the sphere, which touches the cloud of points from outwards.

Remark2: dicretization parameter must be chosen in function of the surface geometry (point density and surface complexity) and according to the required resolution (point number for which the normal must be estimated). 
Remark 3: the method involves the filtering of the point cloud due to the sphere diameter; the sphere can not touch all the points.

Obviously, the determination of the normal for the entire cloud of points generally requires the sphere throwing according to various directions. In this case, the local normal can be calculated several times at the same point. This redundancy is solved by the calculation of a mean value.

\subsection{Visibility Computation}

Generally, the issue of visibility can be treated from two points of view: What is the best view of the part to be machined (one seeks for the best part set-up) or how the part can be seen by the tool (one seeks for the best tool orientation). In both cases, approaches proposed in literature are based on three steps:

- modeling and discretization of the space bounding the object or the object itself,

- identification and choice of adapted mathematical tools

- integration of constraints: collision, tool geometry, accessibility of the machine.

From a machining point of view, literature defines two types of visibility:

- local visibility, which deals with everything concerning the interference between the cutting part of the tool and the surface of the part,

- global visibility, which checks the collision between the tool, the part and the machining environment.

We have classified the various methods of literature in three families. The first family comprises the continuous approaches that do not rely on the space discretization. The most used tool to compute the visibility map is the Gaussian Map (GMap) [11][15]. They are applied to parametric forms such as Bezier surfaces. They reach their limits when the surfaces become very complex. The second group includes methods that use the capabilities of graphics cards [1]. However they require the triangulation of the cloud of points and are therefore not suited to rough clouds of points. The latter family includes methods based on the discretization of space or the polyhedral approximation of the surface to be machined [4]. These methods seem better adapted to complex surfaces and closer to our concern.

\subsubsection{Space Discretization}

After having calculated the normal vector for $\mathrm{n}$ points of the cloud $(\mathrm{n}<\mathrm{N})$, due to the filtering effect of the normal calculation method based on the sphere throwing, the space is discretized. The unit sphere is discretized by the spherical coordinates phi and theta. The values of the discretization of these two angles determine the fineness of the discretization.

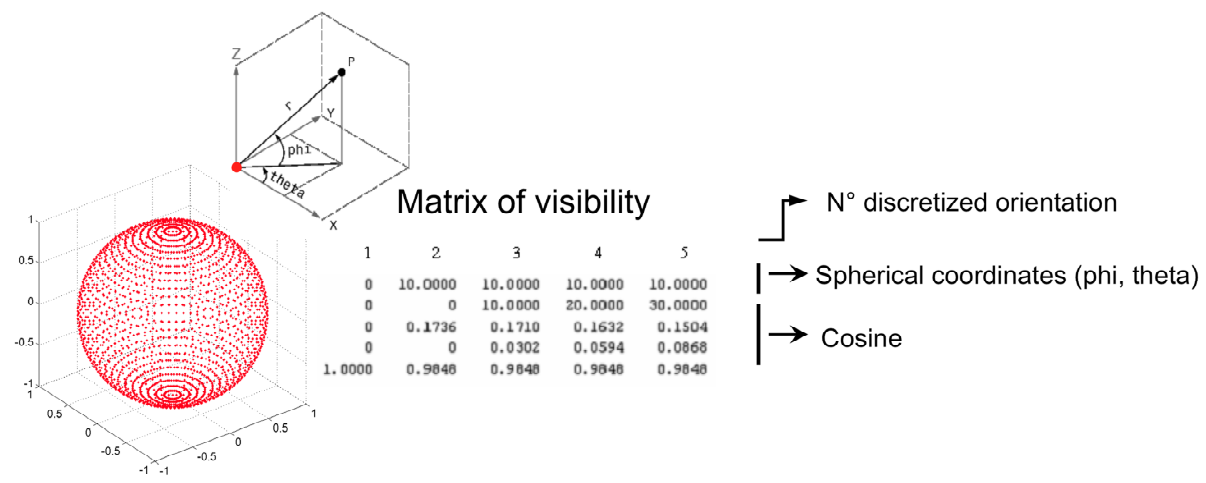

Fig. 3: Space discretization.

\subsubsection{Machine Visibility}

We integrate the geometrical characteristics of the machine and its travel limits to assess the accessibility of the tool at each point of the cloud. Based on the geometrical model of the machine used, we check for each discretized orientation if it belongs or not to the visibility field of the machine. Fig.4 shows the reduction of the number of the acceptable orientations (a) taking into account the 
machine-tool rotary axes travel limits (b) as well as the effective tool length, dimensions of the part holder and of the rough part and translation axis travel limits (c).

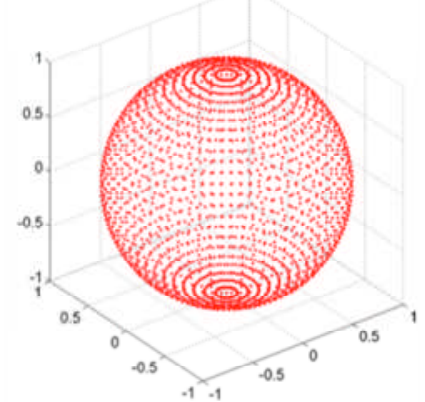

(a) Discretized space

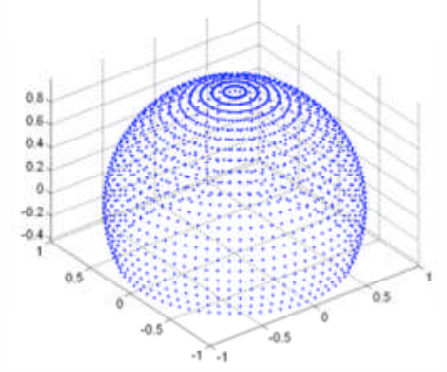

(b) Machine accessibility

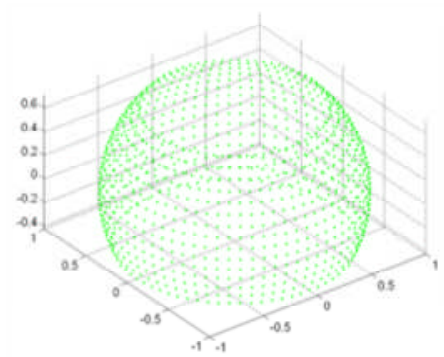

(c) Machine visibility

Fig. 4: Visibility reduction due to the machine tool travels and the tool length.

\subsubsection{Tool Visibility}

After eliminating non-feasible machining directions due to machine visibility, a tool visibility cone is calculated for each of the $\mathrm{n}$ points. The method aims at determining the discretized directions of space which are between two cones whose apex is the concerned point Pi, the axis is the normal vector $\mathrm{N}$ to point $\mathrm{Pi}$ and the angles of the cones are directly related to the position of the contact point between the ball end mill and the considered point. The maximum field of view of a ball end mill is defined between $\mathrm{v} 1=0{ }^{\circ}$ and $\mathrm{v} 2=90$ (Fig. 5).

To ensure the machining quality some technological criteria related to the tool visibility are imposed. Tool visibility is reduced in order to avoid machining with the tool extremity $\left(0^{\circ}<\mathrm{v}<10^{\circ}\right)$ because the cutting speed is close to zero in this case leading to poor surface quality. In order to avoid vertical machining areas where parallel planes machining strategy generates higher scallops on the machined surface, we avoid also the machining with an angle close to $90^{\circ}$ (Fig. 5).

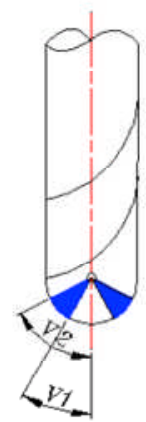

visibilty $: v \in\left[v_{1} ; v_{2}\right]$

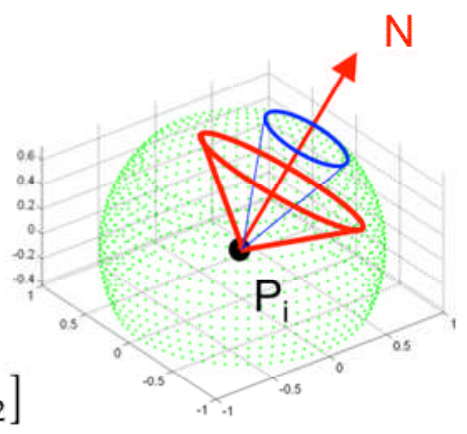

Fig. 5: Tool visibility.

\subsubsection{Global Visibility}

Other constraints affecting the collision between the set tool/tool holder and the set cloud of points/workpiece holder are added. We do not try within this step to test the interference between the cutting part of the tool and the cloud of points. This will be done during tool path computation [7].

Global visibility consists, for any machined point, in testing if any other point of the cloud or of the workpiece holder is inside two cylinders modeling the tool and the tool holder. This calculation is simple but combinatorial and takes most of the full visibility computation time. 


\section{POINT CLOUD SEGMENTATION}

The automatic cloud of point segmentation for area by area machining is a key point of the approach since it must provide all the set-ups allowing the complete surface machining. As presented in the introduction, geometrical deviations on the machined part at the area junctions are inevitable due to re-positioning errors on the machine. The proposed method of automatic segmentation answers the following key question: how may the point cloud be segmented in order to minimize the overlap areas? This problem is expressed through an optimization formulation, with the objective function:

- $\quad$ Find the minimum size of overlapping areas

While satisfying the following constraint functions:

- Machine visibility

- Tool visibility

- Global visibility

- Minimal number of points in each area

- Maximal number of areas

- Overlapping areas forbidden for functional zones

Variables of the optimization problem are:

- The tool orientations (set-ups)

- The number of set-ups

\subsection{Global Segmentation Method}

For a given point of the cloud of points, the global visibility matrix provides all the admissible tool axis orientations respecting the constraint functions relative to visibility. Considering a tool orientation, the global visibility matrix also provides the number of points for which this given orientation is admissible. Therefore, according to the global visibility matrix, points are grouped to respect the following constraint functions:

- The minimal number of points belonging to one area is imposed.

- The number of areas is minimal.

- The overlapping area is minimal.

As the first constraint function is concerned, the minimal number of points is imposed by the user (in function of the total number of points and the maximal number of areas). The direct analysis of the visibility matrix permits to eliminate all the tool orientations that do not satisfy this constraint. The result is a reduced visibility matrix in which remain only the acceptable tool orientations.

Concerning the minimal number of areas, we make the hypothesis that the less the number of areas, the less the overlapping area. Accordingly, we seek for the minimum number of areas that cover almost the entire cloud of points. Let Pa be the acceptable percentage of machined points (for example 95\%). Let $\mathrm{Na}$ be the given number of areas. If a combination of $\mathrm{np}$ orientation among all the admissible orientations, exists that covers the acceptable percentage $\mathrm{Pa}$, then the cloud of points may be machined by using $\mathrm{np}$ set-ups. If such a combination of $\mathrm{np}$ orientations does not exist, $\mathrm{np}$ is incremented until the acceptable percentage is reached, or until the maximum number of orientations required, npmax, is reached (Fig.6). Indeed, the user can choose by advance this number in order to limit tool orientation changes. If this number is reached without finding an admissible combination, the initial part set-up must be changed. Sets of combinations are generated from the reduced visibility matrix using the binomial coefficient.

The complexity of the part and the digitizing noise are source of small areas that are not included in the principal areas. These small isolated areas are integrated to the nearest areas using the Hausdorff distance calculation. However, their integration may cause an alteration of the fidelity to the copy as the tool accessibility criterion is not respected.

Points that are machined by more than one orientation are identified; such points belong to an overlapping area. The number of these points has to be minimal. Indeed, the number of points for which more than one orientation is acceptable is the criterion that is applied to select the set-ups. As a result, the size of the overlapping areas is evaluated by the analysis of the visibility matrix rather than by calculating the effective overlapping areas. The proposed method is very rapid and permits the comparative study of a great number of possible solutions. 


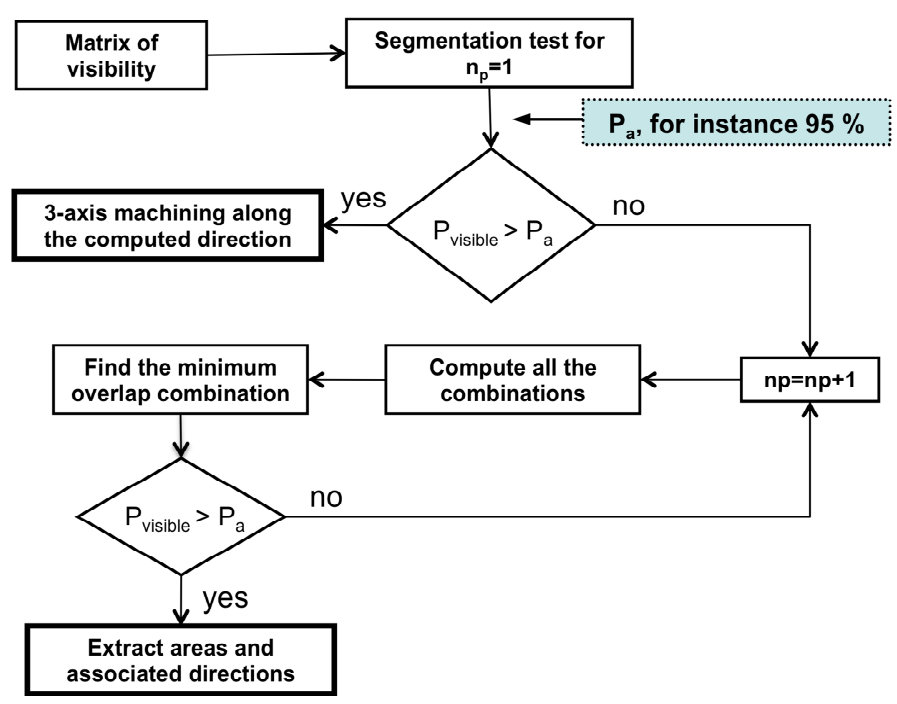

Fig. 6: Global segmentation method.

\subsection{Method Assessment}

In order to assess the proposed approach, a simple use case has been defined. The cloud of points represents a half of sphere mathematically defined. The cloud of points is simulated and consists of 2537 points. The density of the cloud of points is not uniform. The local normal is mathematically determined for each point.

The global visibility matrix is calculated using the Gaussian sphere discretization according the angles phi and theta, which sampling values are respectively phi $=10^{\circ}$ and theta $=30^{\circ}$. The machining is carried out on a 5-axis milling centre Mikron UCP 710. The dicretization provides 216 possible tool axis orientations including machine visibility. The tool diameter is equal to $10 \mathrm{~mm}$ and the tool visibility is defined considering the admissible range $\left[10^{\circ}, 80^{\circ}\right]$.

For this case, the number maximum of orientations required is npmax $=2$. From the matrix of admissible orientations, all the possible combinations of 2 orientations are calculated; this yields to 8778 possible combinations.

Considering an acceptable percentage of machined points at $\mathrm{Pa}=95 \%$, the combination of tool orientations that leads to the minimal overlap is: $\left[\begin{array}{lllll}0.663 & 0.383 & 0.642 ;-0.813 & -0.469 & -0.342\end{array}\right]$. The minimal overlapping area is evaluated here considering the minimal number of points machined with the two orientations. The representation of the two clouds of points corresponding to the two areas is reported in Fig. 7. The first area is represented using green points, and the second area using red points. Remaining points are represented in black. These remaining points are integrated to the nearest area using the Hausdorff distance criterion. The two areas finally obtained as shown in Fig. 7 . The calculation time is estimated to 10 s using Matlab on a Pentium 4.

As shown through this simple use case, the method of segmentation permits:

- $\quad$ To find the minimal numbers of areas,

- To find the segmentation that leads to the minimal overlap. 

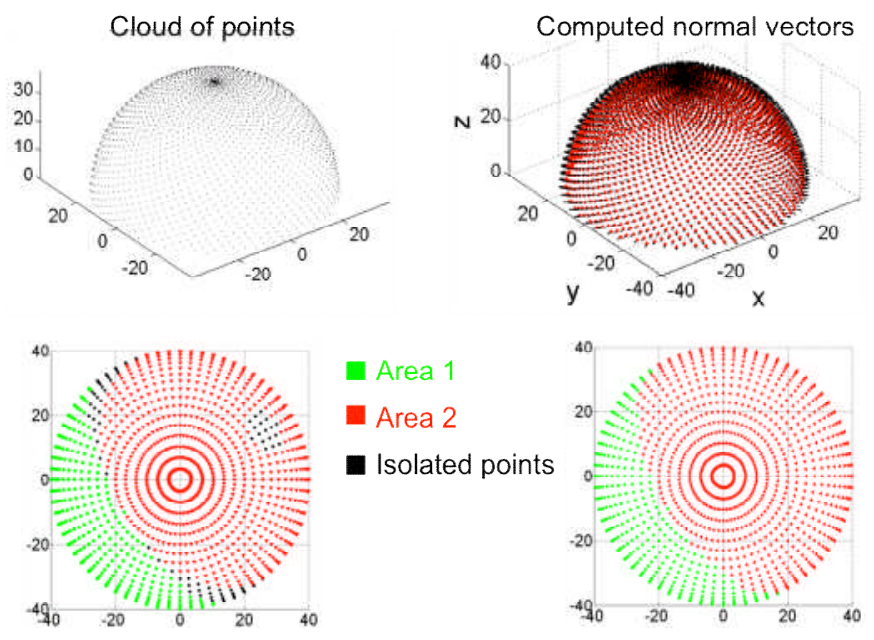

Fig. 7: 2-area segmentation with minimum overlap.

\section{APPLICATION}

In order to test the proposed method for direct object duplication, a realistic use case is proposed. The selected object shown in Fig. 8 is the part of a human femur that constitutes with the pelvis the articulation of the hip. This complex form is an interesting test case to assess our segmentation method, as the complexity of the object shapes requires the use of several set-ups.

All the stages of the duplication chain are detailed next.

\subsection{Acquisition of the Cloud of Points}

The acquisition of the human femur has been carried out using a coordinated measuring machine equipped with a laser plane scanner, Zephyr KZ 50 (http://www.kreon3D.com). The complete object surface capture requires 8 different sensor orientations. The resulting cloud of points is constituted of $1,033,760$ points; the density of points is about 45 points $/ \mathrm{mm}^{2}$.

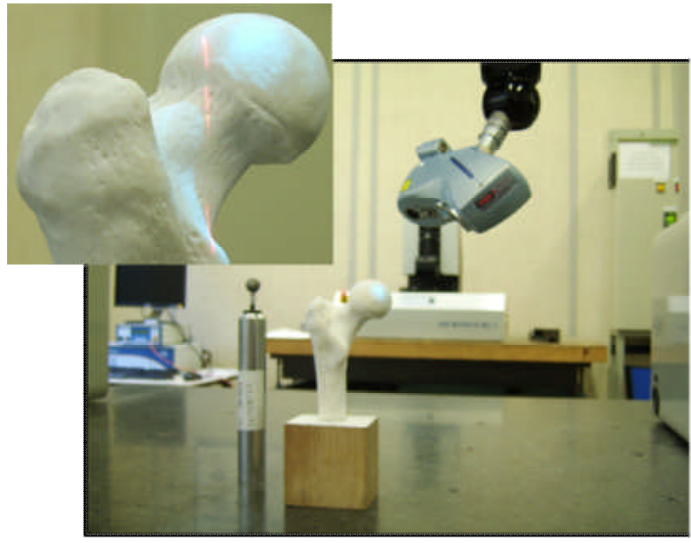

Femur digitizing

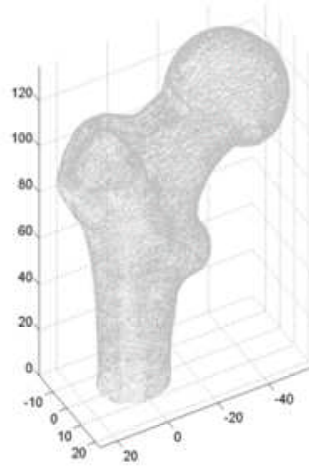

Cloud of points

Fig. 8: Human femur digitizing. 


\subsection{Global Visibility and Cloud Segmentation}

The computation of the normal is performed using a sphere which diameter is $\mathrm{d}=7 \mathrm{~mm}$. Five throwing

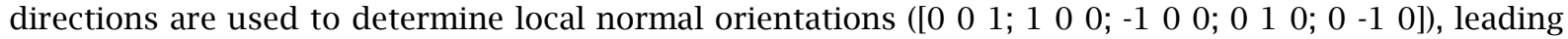
to a lighter cloud of points of 97448 points (only $10 \%$ of the points). This lighten point cloud is further used for global visibility calculation.

The working space discretization is carried out as proposed in the previous section. Considering the machine tool visibility, this leads to 216 possible tool orientations. The tool diameter is equal to $6 \mathrm{~mm}$ and the tool visibility is defined considering the admissible range $\left[10^{\circ}, 60^{\circ}\right]$.

Global visibility includes the avoidance of collisions between the set tool/tool holder and the set point cloud/workpiece/workpiece holder. To carry out the calculation of global visibility, the tool holder and the workpiece holder are described like forbidden clouds of points (Fig. 9).

For the point cloud segmentation, the number of maximum combination is fixed to npmax $=5$, and $\mathrm{Pa}$ is fixed to 95\%. The result of the automatic segmentation is a set of four areas. The four tool axis orientations lead to the machining of $95 \%$ of the cloud of points free from collision. The minimal overlapping area (that represents 18700 points) and the tool orientations are shown in Fig. 9.

1

Digitizing 1033760 points 45 points $/ \mathrm{mm}^{2}$

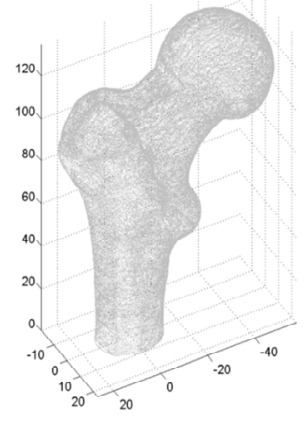

2 Normal computation

$P_{\mathrm{t}} \times \mathrm{P}_{1}=0.5 \times 0.5 \mathrm{~mm} ; \mathrm{D}=7 \mathrm{~mm}$

[0 $01 ; 010 ; 0-10 ; 100 ;-100]$

$\mathrm{T}=126 \mathrm{~min}$

$(\mathrm{n} / \mathrm{N})=97448 / 1033760 ; 9.5 \%$

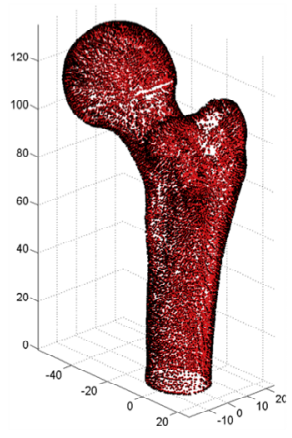

3 Space discretization

$\mathrm{Phi}=10^{\circ}$

Theta $=30^{\circ}$

216 directions

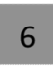
Segmentation 4 areas $\mathrm{Ncomb}=487635$ $\mathrm{T}=45 \mathrm{~min}$ d1 $\quad$ d2 $\quad$ d3 $\begin{array}{llll}0 & 0 & 0.9650 & \mathrm{~d} 4 \\ 0 & -1 & 0\end{array}$ $\begin{array}{lll}0.866 & -0.866 \quad 0\end{array}$ 0.5

$0.5 \quad 0.2588$

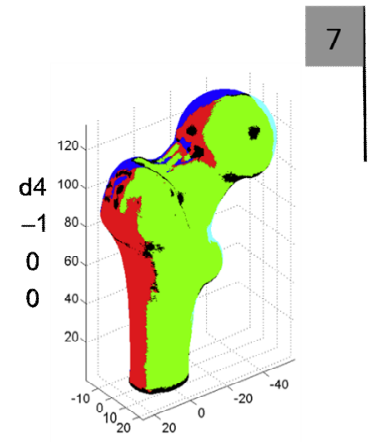

Final Segmentation

After isolated points integration 4 areas

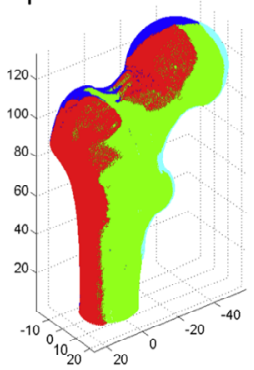

5 Tool and global visibility $v=\left[10^{\circ}-60^{\circ}\right]$ $\mathrm{D}=6 \mathrm{~mm}$ $\mathrm{T}=240 \mathrm{~min}$

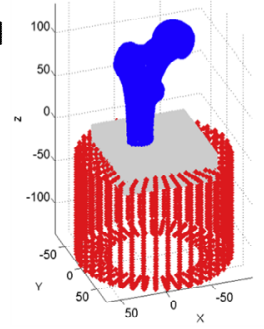

Fig. 9: 5-axis machining segmentation. 


\section{CONCLUSION}

In the paper we have proposed a chain of discrete 5-axis machining of rough clouds of points. This chain can be used in the context of direct duplication of object. Starting from a rough cloud of points collected via optical digitizing means, the main stages are global visibility calculation, data segmentation and actual machining. The global visibility accounts for the tool visibility (local gouging), the machine tool accessibility, and the global visibility (global interferences). Global visibility calculation relies on an original method for local normal estimation on discrete data based on classical method of tool positioning. As the machining is performed area by area, the segmentation method attempts to limit overlapping areas. This criterion is used to define the minimal number of admissible tool orientations. The method is applied with success to the direct duplication of a human femur in a reasonable computation time. Direct duplication could thus be extended to rapid prototyping.

\section{REFERENCES}

[1] Balasubramaniam, M.: Generating 5-axis NC roughing paths directly from a tessellated representation, Computer-Aided Design, 32(4), 2000, pp.261-277.

[2] Breteau, P.; Thiebaut, F.; Bourdet, P.; Lartigue, C.: Towards an approach for rapid copying of freeform surfaces in 5-axis machining, 6th International Conference on Integrated Design and Manufacturing in Mechanical Engineering, Grenoble (France) 2006.

[3] Feng, H-Y.; Teng, Z.: Iso-planar piecewise linear NC tool path generation from discrete measured data points, Computer-Aided Design, 37(1), 2005, 55-64.

[4] Kang J.; Suh J-K.; Machinability and Set-up Orientation for Five-axis Numerically Controlled Machining of Free Surfaces, International Journal of Advanced Manufacturing Technology, 13, 1997, 311-325.

[5] Lin, A-C; Liu, H-T.: Automatic generation of NC cutter path from massive data points, ComputerAided Design, 30(1), 1998, 77-90.

[6] Lorensen, W.; Cline, H.: Marching cubes : A high resolution 3d surface reconstruction algorithm, Proceedings of the 14th annual conference on Computer graphics and interactive techniques, Ed. SIGGRAPH '87. ACM Press, 1987, 163-169.

[7] Makki, M.; Lartigue, C.; Tournier, C.; Thiebaut, F.: Direct duplication of physical models in discrete 5-axis machining, Virtual and Physical Prototyping, 3(2), 2008, 93-103.

[8] Mitra, N., Nguyen, A.: Estimating surface normal in noisy point cloud data, Proceedings of the 19th annual symposium on Computational geometry, San Diego, California, 2003, 322-328.

[9] OuYang, D.; Feng, H.: On the normal vector estimation for point cloud data from smooth surfaces, Computer-Aided Design, 37(10), 2005, 1071-1079.

[10] Park, S-C.; Chung, S-C.: Tool-path generation from measured data, Computer-Aided Design, 35(5), 2003, 467-475.

[11] Tang K., Woo T., Maximum Intersection of Spherical Polygons and Work pièce Orientation for 4 and 5-axis Machining, ASME Journal of Mechanical Design, 114(3), 1989, 477-485.

[12] Teng, Z.; Feng, H-S.; Azeem, A.: generating tool paths from point cloud data via machining area segmentation, International Journal of Advanced Manufacturing Technology, 30, 2006, 254-260.

[13] Varady, T.; Martin, R. R; Cox, J.: Reverse Engineering of geometric models - an introduction. Computer-Aided Design, 29 (4), 1997, 255-268.

[14] Yang, M.; Lee, E.: Segmentation of measured point data using a parametric quadric surface approximation, Computer-Aided Design 31 (7), 1999, 449-457.

[15] Woo, T.: Visibility Maps and Spherical algorithms, Computer-Aided design, 26(1), 1994, 6-16. 